2. Chiotos K, Tamma PD, Gerber JS. Antibiotic stewardship in the intensive care unit: challenges and opportunities. Infect Control Hosp Epidemiol 2019;40:693-698.

3. Taggart LR, Leung E, Muller MP, Matukas LM, Daneman N. Differential outcome of an antimicrobial stewardship audit and feedback program in two intensive care units: a controlled interrupted time series study. BMC Infect Dis 2015;15:480.

4. Morris AM, Bai A, Burry L, et al. Long-term effects of phased implementation of antimicrobial stewardship in academic ICUs: 2007-2015. Crit Care Med 2019;47:159-166.

5. Kaki R, Elligsen M, Walker S, Simor A, Palmay L, Daneman N. Impact of antimicrobial stewardship in critical care: a systematic review. J Antimicrob Chemother 2011;66:1223-1230.

6. Katsios CM, Burry L, Nelson S, et al. An antimicrobial stewardship program improves antimicrobial treatment by culture site and the quality of antimicrobial prescribing in critically ill patients. Crit Care 2012; $16: R 216$.
7. Devchand M, Stewardson AJ, Urbancic KF, et al. Outcomes of an electronic medical record (EMR)-driven intensive care unit (ICU)-antimicrobial stewardship (AMS) ward round: assessing the "Five Moments of Antimicrobial Prescribing." Infect Control Hosp Epidemiol 2019;40:1170-1175.

8. National Antimicrobial Utilisation Surveillance Program (NAUSP). SAHealth website. https:/www.sahealth.sa.gov.au/wps/wcm/connect/public+content/ $\mathrm{sa}+$ health+internet/clinical+resources/clinical+programs + and + practice + guidelines/infection+and+injury+management/antimicrobial+steward ship/national+antimicrobial+utilisation + surveillance + program + nausp/ national+antimicrobial+utilisation + surveillance + program + nausp. Published 2020. Accessed November 9, 2020.

9. James R, Upjohn L, Cotta M, et al. Measuring antimicrobial prescribing quality in Australian hospitals: development and evaluation of a national antimicrobial prescribing survey tool. J Antimicrob Chemother 2015;70:1912-1918.

10. Trubiano JA, Chen C, Cheng AC, Grayson ML, Slavin MA, Thursky KA. Antimicrobial allergy 'labels' drive inappropriate antimicrobial prescribing: lessons for stewardship. J Antimicrob Chemother 2016;71:1715-1722.

\title{
Improved empiric antibiotic prescribing for common infectious disease diagnoses using order sets with built-in clinical decision support in the emergency department
}

\author{
Roslyn M. Seitz MSN, MPH ${ }^{1,2}$ (1) Zanthia Wiley MD³, Christele F. Francois PharmD, BCPS ${ }^{4}$, Tim P. Moran PhD ${ }^{1}$, \\ Jonathan D. Rupp $\mathrm{PhD}^{1}$ and Mary Elizabeth Sexton MD, MSc ${ }^{3}$ \\ ${ }^{1}$ Department of Emergency Medicine, Emory University, Atlanta, Georgia, ${ }^{2}$ Nell Hodgson Woodruff School of Nursing, Emory University, Atlanta, Georgia, ${ }^{3}$ Division \\ of Infectious Diseases, Emory University School of Medicine, Atlanta, Georgia and ${ }^{4}$ Department of Pharmacy, Emory University Hospital, Atlanta, Georgia
}

To the Editor - The Centers for Disease Control and Prevention estimated that in 2018, emergency departments (EDs) generated 12.7 million antibiotic prescriptions. ${ }^{1} \mathrm{Up}$ to $50 \%$ of these prescriptions may have been inappropriate with respect to antibiotic use or selection, dosing, and duration, based on outpatient prescribing estimates. ${ }^{2}$ Improving prescribing is imperative, but historically, EDs are underrepresented in antibiotic stewardship studies. ${ }^{4}$ EDs may benefit from implementation of the recommended components of an antimicrobial stewardship program, including decision-making tools based on facility-specific practice guidelines. ${ }^{3}$ For example, antibiotic order sets within an electronic medical record (EMR) have been shown to improve adherence to evidence-based prescribing for single diagnoses, ${ }^{5,6}$ although the use of multiple order sets for a variety of diagnoses has not been well studied. We implemented EMR order sets for common infectious diagnoses in the ED, compared the prescribing practices of providers who utilized them to those who did not, and surveyed providers for barriers to use.

\section{Methods}

This study was part of a larger intervention to improve antibiotic prescribing and reduce Clostridioides difficile infection (CDI) at a 500 -bed quaternary-care academic medical center with $\sim 50,500$

Author for correspondence: Roslyn M. Seitz, E-mail: Roslyn.seitz@emoryhealthcare.org Cite this article: Seitz RM, et al. (2022). Improved empiric antibiotic prescribing for common infectious disease diagnoses using order sets with built-in clinical decision support in the emergency department. Infection Control \& Hospital Epidemiology, 43: 672-674, https://doi.org/10.1017/ice.2021.73 yearly ED visits. Order sets were created for cystitis, pyelonephritis, pneumonia, chronic obstructive pulmonary disease (COPD), and cellulitis that included recommended antibiotics and first dose in the ED, followed by a prepopulated prescription for an appropriate duration. Antibiotic choices were prioritized based on clinical practice guidelines, ${ }^{7-9}$ the hospital antibiogram, and a desire to avoid antibiotics associated with higher CDI risk (eg, fluoroquinolones), with guidance included for dosing in patients with renal impairment.

The order sets were deployed in March 2019, with clinician education via a presentation ( $40 \%$ attendance), 1 -on- 1 sessions $(60 \%$ of clinicians), and 3 informational e-mails. A survey adapted from Vandenberg et $\mathrm{al}^{10}$ was sent to all ED clinicians in November 2019 to assess whether the order sets were being used and whether they were beneficial to their practice.

Additionally, a retrospective chart review was conducted from October 1, 2019, to November 1, 2019, to assess the impact on prescribing practices for patients presenting with 1 of the 5 diagnoses with a corresponding order set, identified using International Classification of Disease, Tenth Revision (ICD-10) codes. Charts were manually reviewed for whether an order set was used, antibiotic doses given in the $\mathrm{ED}$, antibiotic prescribed, creatinine clearance, special population status (eg, pregnancy or organ transplant), prior culture data, and whether a subspecialty consultation was obtained. Patients were excluded from the analysis if antibiotics were not prescribed, if they belonged to a special population, or if they received subspecialty consultation. In total, 213 charts were reviewed and 104 met inclusion criteria. Encounters with order-set use were compared to those without order-set use for appropriate 
Table 1. Appropriate Antibiotic Prescribing With and Without Order Set Use

\begin{tabular}{|c|c|c|c|}
\hline Variable & With Order Set Use & Without Order Set Use & $P$ Value \\
\hline First dose of antibiotic given in the ED $(n=104)$ & $90.9 \%(20 / 22)$ & $71.2 \%(54 / 82)$ & .02 \\
\hline Appropriate empiric agent selected without prior culture data or allergy considerations $(n=99)$ & $86.4 \%(19 / 22)$ & $33.8 \%(26 / 77)$ & $<.001$ \\
\hline Optimal duration of therapy prescribed & $68.2 \%(15 / 22)$ & $24.5 \%(13 / 53)$ & .0004 \\
\hline Antibiotic prescription with inappropriate renal dosing & $10 \%(2 / 20)$ & $3 \%(2 / 72)$ & .43 \\
\hline
\end{tabular}

Note. ED, emergency department.

antibiotic selection, duration, and renal dosing. We used the $\chi^{2}$ test with a $P<.05$ significance level and OpenEpi Open Source Epidemiologic Statistics for Public Health version 3.01 software (www.openepi.com). Patients who received an inappropriate antibiotic were excluded when comparing durations of therapy, and those without a measured creatinine level were excluded when comparing appropriate renal dosing. The study was approved by the Emory Institutional Review Board.

\section{Results}

\section{Provider survey}

The overall response rate was $59 \%$. Just more than half $(51.6 \%)$ of clinicians were physicians and $48.4 \%$ were APPs. Most respondents were aware of the order sets $(75.0 \%)$ and $59.4 \%$ reported using them. Of the clinicians who used the order sets, $78.9 \%$ reported that they saved time, $84.2 \%$ reported ease of use, and $94.7 \%$ reported successful use.

\section{Chart review}

Order sets were used in 22 (21\%) of 104 qualifying patient encounters. Use varied by diagnosis. An order set was used in 12 (43.8\%) of 32 patients with uncomplicated cystitis, $2(22.2 \%)$ of 9 patients with pyelonephritis, 2 (20\%) of 10 patients with cellulitis, 3 $(6.7 \%)$ of 45 patients with complicated cystitis, and none of 8 patients with pneumonia or COPD. Patients were more likely to receive the first antibiotic dose in the ED when order sets were used $(P=.02)$ (Table 1). Patients were also more likely to receive an appropriate antibiotic $(P<.001)$ and to have an appropriate duration prescribed $(P=.0004)$ when order sets were used. We did not detect a statistically significant difference in appropriate renal dosing between the 2 groups.

\section{Discussion}

In this study, EMR antibiotic order sets for treatment of common infectious syndromes were implemented in the ED. They were only used in $21 \%$ of qualifying patients, but in these cases they were associated with improved antibiotic selection, first-dose timing, and prescription duration. Almost all providers who reported utilizing the order sets noted that they were easy to use and saved time.

Given the advantages of order-set use, improving uptake will be a focus of future interventions. Historically, order sets have been evaluated for single conditions, ${ }^{5}$ but availability of multiple order sets may improve provider acceptance. We observed that clinicians used order sets for uncomplicated cystitis more frequently than for other conditions, which may be related to the frequency of diagnosis and a large number of antibiotic choices. Ensuring that providers who used the cystitis order set are aware of the other options and can educate other clinicians may help to increase acceptance.

Larger studies are needed to further evaluate the impact of order sets on prescribing and patient care outcomes because early data indicate that they may improve antimicrobial stewardship. In the future, we plan (1) to expand order-set offerings within the ED and in outpatient settings; (2) to increase use via additional didactics, peer-to-peer education, audit and feedback; and (3) to evaluate trends in antibiotic prescribing over a longer time period.

Acknowledgments. We thank K. Ashley Jones, PharmD, BCIDP, for assistance related to this study.

Financial support. This study was supported by funding from the Centers for Disease Control and Prevention's Investments to Combat Antibiotic Resistance (grant no. 200-2016-91944).

Conflicts of interest. All authors report no conflicts of interest relevant to this study.

\section{References}

1. Outpatient antibiotic prescriptions-United States, 2018. Centers for Disease Control and Prevention website. https://www.cdc.gov/antibioticuse/community/programs-measurement/state-local-activities/outpatient-anti biotic-prescriptions-US-2018.html. Published 2018. Accessed on November 21, 2020.

2. Antibiotic resistance threats in the United States 2019. Centers for Disease Control and Prevention website. https://www.cdc.gov/drugresistance/pdf/ threats-report/2019-ar-threats-report-508.pdf. Published 2019. Accessed on November 21, 2020.

3. Barlam TF, Cosgrove SE, Abbo LM, et al. Implementing an antimicrobial stewardship program: guidelines by the Infectious Disease Society of America and the Society for Healthcare Epidemiology. Clin Infect Dis 2016;62(10)e51-e77.

4. Pulia M, Redwood R, May L. Antimicrobial stewardship in the emergency department. Emerg Med Clin North Am 2018;36:853-872.

5. Heckler MT, Fox CJ, Son AH, et al. Effect of a stewardship intervention on adherence to uncomplicated cystitis and pyelonephritis guidelines in an emergency department setting. PloS One 104;9(2):e87899.

6. Forest GN, van Schooneveld TC, Kullar R, et al. Use of electronic health records and clinical decision support systems for antimicrobial stewardship. Clin Infect Dis 2014;59 suppl 3:S122-S133.

7. Gupta K, Hooton TM, Naber KG, et al. International clinical practice guidelines for the treatment of acute uncomplicated cystitis and pyelonephritis in women: a 2010 update by the Infectious Diseases Society of America and the European Society for Microbiology and Infectious Diseases. Clin Infect Dis 2011;52(5):e103-e120.

8. Metlay JP, Waterer GW, Long AC, et al. Diagnosis and treatment of adults with community-acquired pneumonia. an official clinical practice guideline 
of the American Thoracic Society and Infectious Diseases Society of America. Am J Respir Crit Care Med 2019;200(7):e45-e67.

9. GOLD Global Strategy for the Diagnosis, Management and Prevention of COPD. Global Initiative for Chronic Obstructive Lung Disease (GOLD) website. https://goldcopd.org/wp-content/uploads/2017/11/GOLD-2018-
v6.0-FINAL-revised-20-Nov_WMS.pdf. Published 2018. Accessed November 29, 2020.

10. Vandenberg AE, Vaughan CP, Stevens $\mathrm{M}$, et al. Improving geriatric prescribing in the ED: a qualitative study of facilitators and barriers to clinical decision support tool use. Int J Qual Health Care 2017 Feb 1;29(1):117-123.

\title{
Can intravenous antimicrobial start data reported to the National Healthcare Safety Network determine appropriateness of antibiotic use in hemodialysis patients?
}

\author{
Priti R. Patel MD, $\mathrm{MPH}^{1}{ }^{(1)}$, Shannon A. Novosad MD, $\mathrm{MPH}^{1}$ and Ibironke W. Apata $\mathrm{MD}^{1,2}$ \\ ${ }^{1}$ Centers for Disease Control and Prevention, Atlanta, Georgia and ${ }^{2}$ Emory University School of Medicine, Atlanta, Georgia
}

To the Editor-In their article, "Inappropriate intravenous antimicrobial starts: An antimicrobial stewardship metric for hemodialysis facilities," Hahn et al $^{1}$ describe their application of data reported to CDC's National Healthcare Safety Network (NHSN) to determine appropriateness of IV antibiotic use in outpatient hemodialysis centers. NHSN's Dialysis Event (DE) surveillance system was designed to track bloodstream infections (BSIs) and other vascular access infections in hemodialysis outpatients through monitoring of events such as positive blood cultures. The authors examined outpatient IV antimicrobial start (IVAS) events reported to NHSN and considered any IVAS without documentation of coreported positive blood culture, collection of blood sample for culture, or local access site infection to be inappropriate, even when symptoms such as fever, chills, rigors, or drop in blood pressure were present. We applaud these investigators for drawing necessary attention to the issue of antibiotic use in dialysis patients, which is an important area of study with limited data, and for exploring the use of data to inform improvement in practice. However, we have concerns about their approach to the categorization of antibiotic use without incorporation of relevant clinical information or validation of NHSN data for this purpose, and the potential for unintended consequences among patients at high risk for infections and sepsis.

Intravenous antimicrobial starts largely represent empiric antibiotic doses for suspected infection. Initiating empiric antibiotics in the presence of signs and symptoms of serious infection, while awaiting culture information, should be considered appropriate use. ${ }^{2}$ However, Hahn et al. excluded symptom data from their assessment of appropriateness, even for the 260 IVAS events (27\%) for which these data were available in NHSN. They explained that the data were not consistently reported. We agree that gaps exist in the completeness of the symptom information, which is one of several inherent limitations with the use of NHSN data in this manner. The IVAS events that occurred in the context of symptoms but without preceding blood culture collection may represent gaps in documentation (of blood cultures) or adherence to blood culture collection protocols, rather than actual inappropriate antibiotic use. A more robust appropriateness

\footnotetext{
Author for correspondence: Priti Patel, E-mail: ppatel@cdc.gov

Cite this article: Patel PR, Novosad SA, and Apata IW. (2022). Can intravenous antimicrobial start data reported to the National Healthcare Safety Network determine appropriateness of antibiotic use in hemodialysis patients?. Infection Control \& Hospital Epidemiology, 43: 674-675, https://doi.org/10.1017/ice.2021.55
}

determination should incorporate medical record review to identify symptoms that might have been unreported to NHSN, and other relevant information.

Hahn et al acknowledge that they did not attempt to identify related events in the same patient (that might justify the IVAS) if they were not coreported on the same form. The NHSN does not require that events such as positive blood cultures and IVAS be coreported as long as each event is reported. Unique identifiers exist within NHSN to facilitate linkage of events that occurred in the same patient, even when events are not submitted together. ${ }^{3}$ Other potential justifications for antibiotic administration, such as surgical prophylaxis (for which blood culture collection is not typically warranted prior to antibiotic administration), are not captured within the dialysis event surveillance; thus, they were not factored into the appropriateness determination.

Previous studies have identified de-escalation of antibiotic therapy as the most common missed opportunity for improved antibiotic use among patients on hemodialysis, ${ }^{4,5}$ suggesting that continued antibiotic doses, as opposed to the initial dose, should be the priority for antibiotic use measurement efforts in this setting. However, the NHSN does not capture all IV antibiotic doses administered to dialysis patients, nor the specific antibiotic administered (other than vancomycin starts). In our opinion, expanded efforts are needed to identify stewardship interventions that could result in meaningful improvements in outcomes in this population, to help guide the development of useful metrics.

Hahn et al conclude that $57.5 \%$ of all IVAS in the facilities examined were inappropriate. Given the aforementioned challenges, we believe that they may have overestimated true inappropriate prescribing of IV antibiotics in dialysis facilities. Prior studies have demonstrated that initial doses of antibiotic treatment are a small proportion of all antibiotic doses received by hemodialysis outpatients (eg, 7.6\% in one study). ${ }^{4,5}$ In previous studies of maintenance hemodialysis patients, $10 \%-43 \%$ of initial IV antibiotic treatment doses were classified as inappropriate, ${ }^{4,5}$ and $20 \%-30 \%$ of all vancomycin or IV antibiotic doses prescribed (including continued doses) were classified as inappropriate. ${ }^{5,6}$ Few studies have evaluated antibiotic use in outpatient hemodialysis centers, and the resultant estimates of inappropriate use are often challenged by limited medical record documentation. ${ }^{4,5,7}$ Hahn et al also draw parallels between outpatient hemodialysis care and outpatient primary care settings. However, hemodialysis clinics and patients are unlike most general outpatient 